\title{
Irradiation of rabbit retina with diode and Nd:YAG lasers
}

Dominic McHugh, Ceri England, Eugen van der Zypen, John Marshall, Franz Fankhauser, Sylwia Fankhauser-Kwasnieska

\begin{abstract}
Aims-This study was carried out to compare the effects of continuous wave infrared laser radiation on pigmented and albino rabbit retinas at two wavelengths: $810 \mathrm{~nm}$ (diode) and $1064 \mathrm{~nm}$ (Nd:YAG).

Methods-Transpupillary laser pulses were applied with a spot size of $200 \mu \mathrm{m}$ and durations of $200 \mathrm{~ms}$ (pigmented rabbits) and 0.5-1 s (albino rabbits). Light and electron microscopic analyses were performed immediately after exposure.

Results-In pigmented rabbits, threshold lesions were induced using a power of $100 \mathrm{~mW}$ with the diode and $200 \mathrm{~mW}$ with the Nd:YAG lasers. Damage was incurred by the retinal pigment epithelium with extension into the superficial and mid choroid posteriorly and into the outer retina anteriorly. In albino rabbits, lesions of comparable anteroposterior extension were identified using a power of $10 \mathrm{~W}$ with the Nd:YAG laser. Using diode laser irradiation, a maximum power output of $1.2 \mathrm{~W}$ failed to produce discernible lesions.

Conclusions-The observed patterns of morphological damage are produced by complex tissue radiation interactions. In pigmented animals, this was primarily related to absorption of radiant energy by melanin within the retinal pigment epithelium and the choroidal melanocytes. In albino rabbits, laser induced effects occurred as a consequence of multiple scattering, together with absorption within haemoglobin and possibly also within tissue water. The data obtained provide further insight into the biological mechanisms arising from retinal photocoagulation with near infrared lasers.
\end{abstract}

(Br f Ophthalmol 1995; 79: 672-677)

Photocoagulation has been employed in the treatment of retinal conditions for over 40 years. Meyer-Schwickerath initially used focused sunlight and subsequently the xenon arc photocoagulator, which provided a source of broad band optical radiation and successfully produced full thickness chorioretinal lesions. ${ }^{1}$ Xenon arc devices are of proved effectiveness in the treatment of proliferative diabetic retinopathy ${ }^{2}$ and are still commonly used in retinal therapy.

After its introduction in $1960,{ }^{3}$ the ruby laser (emitting at $694.3 \mathrm{~nm}$ ) aroused interest among ophthalmologists. Although it was reasonably successful in producing chorioretinal adhesions in the treatment of retinal breaks, results obtained in treating retinal vascular conditions were disappointing. ${ }^{4}$

Lasers emitting at other wavelengths were subsequently developed and adapted for use in ophthalmology. These include the argon $(488-515 \cdot 5 \mathrm{~nm})$, the krypton red $(647 \mathrm{~nm})$, and the tunable dye lasers. Many studies have demonstrated their efficacy in the treatment of common retinal conditions, such as proliferative diabetic retinopathy, ${ }^{5}$ forms of diabetic maculopathy, ${ }^{6}$ the complications ensuing from retinal vein thrombosis, ${ }^{7}$ and subretinal neovascular membranes. ${ }^{8}$

Although argon, krypton, and dye lasers have different emission wavelengths their effects are broadly similar in terms of both retinal damage and therapeutic efficacy. These observations lend support to the view that wavelength is relatively unimportant as a determinant of clinical response. The advent of solid state continuous wave diode $(810 \mathrm{~nm})$ and Nd:YAG (1064 $\mathrm{nm}$ ) lasers allows this concept to be tested in relation to tissue irradiation with near infrared energy. Analysis of diode and Nd:YAG laser retinal lesions in pilot studies have indicated that they are similar to those produced by conventional clinical photocoagulators and in particular to those induced using krypton lasers. ${ }^{9-12}$ Initial clinical studies have also demonstrated comparable results with earlier trials employing argon and krypton lasers. ${ }^{13} 14$

The aim of the current study was to compare the morphological effects of continuous wave diode and Nd:YAG irradiation on rabbit retina, thereby gaining a clearer insight into the thermal damage processes induced after laser tissue interactions. It was anticipated that the information obtained would help to optimise treatment variables for performing near infrared laser retinal photocoagulation. 

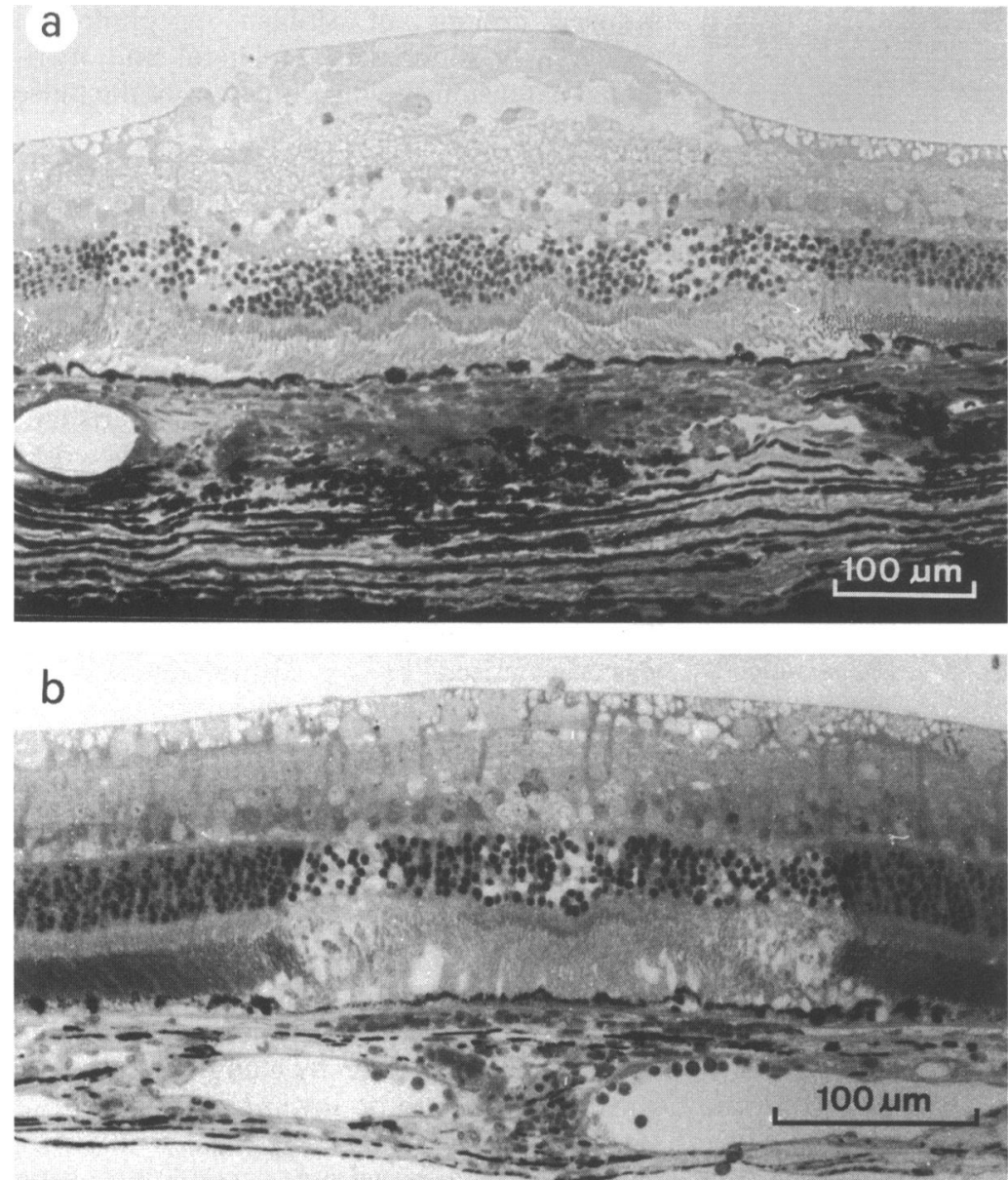

Figure 1 Light micrographs of acute retinal lesions produced in pigmented rabbits using (a) diode (power $250 \mathrm{~mW}$; pulse duration $200 \mathrm{~ms}$ ) and (b) $N d: Y A G$ (power $800 \mathrm{~mW}$; pulse duration $200 \mathrm{~ms}$ ) laser radiation. Each impact is sharply defined laterally, the extension in this direction depending on the pulse power employed and is characterised by a central, strongly coagulated region exhibiting minimal structural distortion and peripheral zones manifesting predominantly swelling and disruptive phenomena. In the meridional direction, strong coagulative effects also give way to swelling and disruptive ones, the degree of inner extension depending upon the pulse power used. In (b), these latter phenomena commence at, and are limited predominantly to, the outer nuclear layer which exhibits a reduction in nuclei density; whereas in (a), they begin at the border between the outer nuclear and outer plexiform layers and extend to the inner limiting membrane, thus giving rise to a prominent inner buckling of the sensory retina. Semi-thin sections stained with toluidine blue. Negative magnifications: (a) $\times 26$; (b) $\times 39$.

\section{Materials and methods}

\section{LASERS}

The diode laser used in these studies was a portable device (Microlase, Keeler Holdings Plc, Windsor, UK), which could be mounted on a Haag-Streit slit-lamp microscope (HaagStreit, Bern, Switzerland). The cone angle of the treatment beam was 14 degrees; the aiming beam was furnished by a second diode laser source (wavelength $670 \mathrm{~nm}$ ). Selected spot size was $200 \mu \mathrm{m}$ and pulse duration $200 \mathrm{~ms}$ (pigmented rabbits), or $990 \mathrm{~ms}$ (albino rabbits). The maximum available power was $1 \cdot 2 \mathrm{~W}$.

A Nd:YAG laser operating in the continuous wave mode was employed (MR3 Meridian, Bern, Switzerland). The cone angle of the treatment beam was 9 degrees and aiming was accomplished with a helium neon laser (wavelength $632 \mathrm{~nm}$ ). Selected spot size was $200 \mu \mathrm{m}$, and pulse duration $200 \mathrm{~ms}$ (pigmented rabbits) or $0 \cdot 5-1 \mathrm{~s} \mathrm{(albino}$ rabbits). The maximum available power was $10 \mathrm{~W}$.

\section{RABBITS}

All procedures were conducted in compliance with the ARVO statement for the use of animals in ophthalmic and vision research. General anaesthesia (Rompun-Ketalar) was induced in four pigmented and two albino rabbits. Pupillary mydriasis was induced using cyclopentolate drops $1 \%$. The retina of one eye in each animal was irradiated through a planoconcave Goldmann contact lens with either the Nd:YAG or diode lasers. The energy required to produce a visible reaction was determined in preliminary experiments by increasing pulse power in $100 \mathrm{~mW}$ increments from subthreshold values. Impacts were delivered to the mid-peripheral region of the retina inferior to the myelinated fibre layer. The power was increased in $50 \mathrm{~mW}$ (diode), or 200-300 mW (Nd:YAG) increments to produce progressively more intense lesions.

TISSUE PREPARATION

Animals were killed by an overdose of sodium pentobarbitone immediately after irradiation. They were then perfused via the aorta, initially with Ringer's solution (containing procaine hydrochloride $(5 \mathrm{~g} / \mathrm{l})$ and heparin $(25000 \mathrm{U} / \mathrm{l})$ ) at $37^{\circ} \mathrm{C}$ and then with $2.5 \% \quad(\mathrm{v} / \mathrm{v})$ glutaraldehyde solution (in $90 \mathrm{mM}$ phosphate buffer, pH 7.4, 350 mosmol) at $4^{\circ} \mathrm{C}$. Perfusion pressure was maintained within the range 115-120 $\mathrm{mm} \mathrm{Hg}$.

The eyes were enucleated and transferred to glutaraldehyde solution (as above), within which they were divided into anterior and posterior segments by cutting slightly posterior to the ora serrata. After fixation overnight at $4^{\circ} \mathrm{C}$, lesions were photographed with a Wild M400 biomicroscope. Impact sites were further dissected for light (LM) and transmission electron (TEM) microscopy. Tissue blocks were washed three times in 90

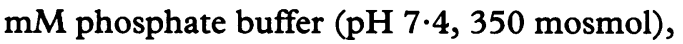
postfixed in $2 \%(\mathrm{w} / \mathrm{v})$ osmium tetroxide solution for 3 hours, stained with uranyl acetate $(0.5 \%(\mathrm{w} / \mathrm{v})$ in $50 \mathrm{mM}$ maleate buffer), and then dehydrated in a graded series of increasing ethanol concentration before embedding in Epon. Sections were cut on a Reichert-Jung Ultracut E, ultramicrotome. Semi-thin $(\sim 3-4 \mu \mathrm{m})$ sections were photographed in a Vanox photomicroscope; ultrathin sections $(\sim 70 \mathrm{~nm})$ were stained with $7 \%(w / v)$ lead citrate and $6 \%(w / v)$ uranyl acetate, and examined in a Philips EM-300 electron microscope.

\section{Results}

\section{ACUTE LESIONS}

\section{Ophthalmoscopic/biomicroscopic appearances}

Pigmented rabbits. Ophthalmoscopically visible lesions were produced using powers of $100 \mathrm{~mW}$ (diode laser) and $200 \mathrm{~mW}$ (Nd:YAG laser) and a pulse duration of $200 \mathrm{~ms}$. More intense effects were realised with each laser using higher pulse powers. 


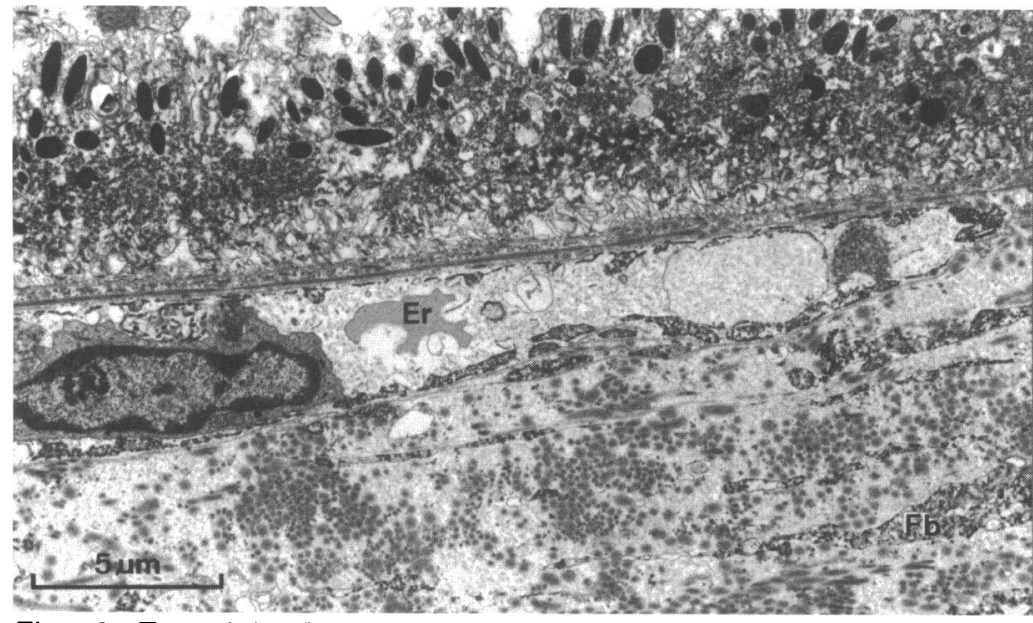

Figure 2 Transmission electron micrograph depicting pigmented retinal epithelium and inner choroid of impact centre after irradiation with the diode laser (power $250 \mathrm{~mW}$; pulse duration 200 ms; acute lesion). Bruch's membrane remains intact, although constituent collagen fibrils exhibit signs of thermal damage (increased cross sectional diameter). The endothelium of the choriocapillaris illustrated has undergone coagulation; within the vessel lumen, heat precipitated proteins and damaged erythrocytes (Er) are to be seen. In the stroma, both normal and thermally damaged collagen fibrils are apparent. $F b=$ coagulated cytoplasmic process of a fibroblast. Negative magnification: $\times 2175$.

Albino rabbits. Nd:YAG laser radiation produced clearly visible lesions, with an altered light reflex at a power of $8 \mathrm{~W}$ and an exposure duration of $500 \mathrm{~ms}$; a more pronounced reaction was realised using a $10 \mathrm{~W} / 1 \mathrm{~s}$ combination. The diode laser failed to produce either a visible response or any light microscopic change at its maximum power output $(1 \cdot 2 \mathrm{~W})$.

\section{Microscopy}

Pigmented rabbits. At the light microscopic level, diode and $\mathrm{Nd}: \mathrm{YAG}$ laser radiation induced lesions of similar morphological appearance, differences in lateral and meridional extensions being a function of the pulse power employed.

Lesions were sharply defined laterally (Fig 1), and in sections traversing the impact core two zones could be distinguished. At the centre, the tissue showed the least structural disturbance. At low pulse powers, this effect extended from the pigmented retinal epithelium to the inner segments of photoreceptor cells, whereas at higher powers their perikarya were also included, as evidenced by the compact appearance of the outer nuclear layer (Fig 1a). A characteristic band of enhanced staining affinity at the level of inner segments (Fig 1b) denoted intense thermal damage. The inner boundary of coagulated tissue was recognised by an abrupt dislocation, decreased compactness, and increased structural disturbance of the adjacent layers, since regions which had incurred minimal thermal insult were more susceptible to secondary swelling and distortion during the post-irradiation period. At low pulse powers, this effect was limited principally to the outer nuclear layer, whereas at higher powers it commenced at the border between outer nuclear and outer plexiform layers and extended to a variable degree inwards. The swelling thereby incurred gave rise to an inward buckling of the sensory retina (Fig 1a). External to the pigmented retinal epithelium, coagulation of the choriocapillaris was observed and the lateral extension of this response corresponded well with the lesion borders in the retinal pigment epithelium (Fig 1b). Coagulative effect $\underline{m}$

过

$\overrightarrow{0}$

क्ष

夏

ข้

ㄱำ

?

요

c

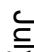

$\overrightarrow{8}$

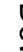

क्ष

음

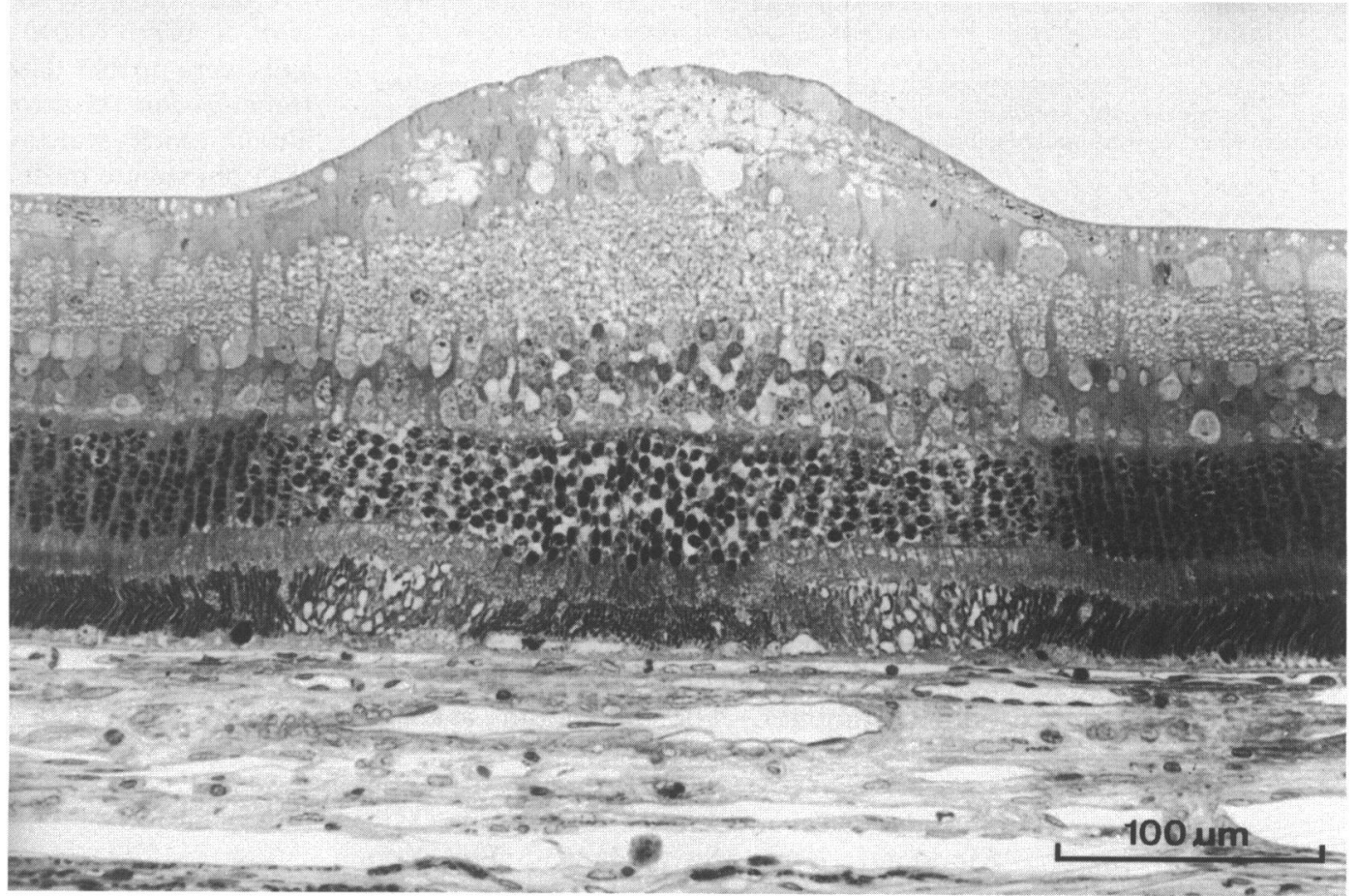

Figure 3 Light micrograph of acute retinal lesion produced in albino rabbit using Nd:YAG laser radiation (power $8 W$; pulse duration $500 \mathrm{~ms}$ ). At the impact centre, photoreceptor cell outer and inner segments are strongly coagulated, as indicated by the minimal structural disturbance manifested in these layers. The outer nuclear layer exhibits both thermal (intensely stained nuclei) and swelling phenomena (reduction in nuclei density); strata further inwards reveal predominantly the latter effects, thus giving rise to buckling. In the lateral direction, peripheral regions also manifest predominantly swelling/disruptive phenomena; note the severe structural disturbance incurred at the levels of photoreceptor cell outer and inner segments. Within the choriocapillaris, no signs of blood flow stasis are apparent. Semi-thin section stained with toluidine blue. Negative magnification: $\times 55$. 


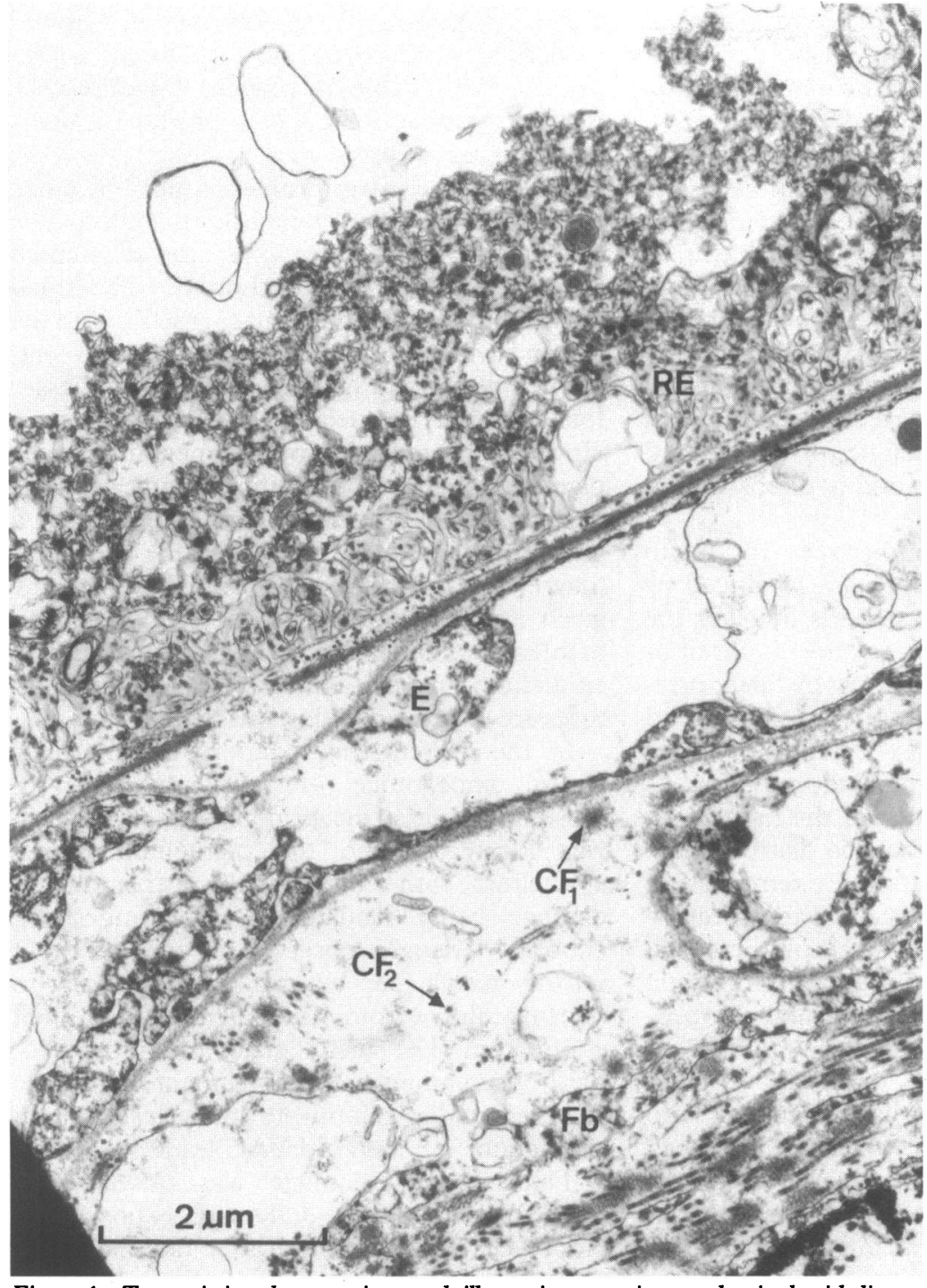

Figure 4 Transmission electron micrograph illustrating non-pigmented retinal epithelium (RE) and inner choroid of albino rabbit after irradiation with the Nd:YAG laser (power 10 W; pulse duration 500 ms; acute lesion). Non-pigmented retinal epithelial cells exhibit an intensely coagulated cytoplasm, but apical extensions have been disrupted; basal processes retain their intimacy with the basal lamina which is unbroken and remains in situ. Within the illustrated choriocapillaris, endothelial cells (E) are coagulated, but there are no signs of blood flow stasis within the vessel lumen. Note that the epithelial facing aspect of the endothelium exhibits disruption and its basal lamina luminal bowing at the position where a dehiscence in the elastic lamina of Bruch's membrane is manifested. Both thermally damaged $\left(C F_{1}\right)$ and normal $\left(C F_{2}\right)$ collagen fibrils are apparent in the surrounding stroma. $F b=$ coagulated cytoplasmic process of a fibroblast. Negative magnification $\times 7050$.
Table 1 Approximate percentages of transmission and absorption for diode and $N d: Y A G$ laser retinal photocoagulation in rabbits

\begin{tabular}{|c|c|c|}
\hline & $\begin{array}{l}\text { Diode } \\
810 \mathrm{~nm}\end{array}$ & $\begin{array}{l}N d: Y A G \\
1064 \mathrm{~nm}\end{array}$ \\
\hline $\begin{array}{l}\text { Transmission (\%) } \\
\text { Absorption (\%) }\end{array}$ & 98 & 70 \\
\hline $\begin{array}{l}\text { Melanin }{ }^{\star} \\
\text { Melanin } \dagger \\
\text { Oxyhaemoglobin } \\
\text { Reduced haemoglobin } \\
\text { Water }\left(\mathrm{cm}^{-1}\right)^{35}\end{array}$ & $\begin{array}{l}35 \\
7 \\
8 \\
8 \\
0 \cdot 4\end{array}$ & $\begin{array}{c}12 \\
2 \\
8 \\
<1 \\
0.04\end{array}$ \\
\hline
\end{tabular}

^Retinal pigment epithelium and choroid.

†Retinal pigment epithelium alone. ${ }^{26}$

cytoplasm exhibited the granulation effect typical of coagulated cells. Within capillary lumens, signs of coagulation were evinced by their fine floccular appearance, this manifestation being engendered by heat induced precipitation of plasma proteins.

Albino rabbits. Similar phenomena to those described for pigmented rabbits were observed in albino animals using Nd:YAG laser radiation. A notable exception was the choriocapillaris, which exhibited no signs of blood flow stasis (Fig 3).

Ultrastructural changes to the non-pigmented retinal epithelium (Fig 4) were comparable with those described for pigmented rabbits. Within the choroid, thermal damage was incurred both by stromal components (Fig 4) and vascular endothelial cells, but signs of blood flow stasis were not manifested.

\section{Discussion}

This study represents the first direct comparison of retinal lesion morphology after irradiation with continuous wave diode and Nd:YAG laser. Radiation from each laser induced impacts of comparable appearance in pigmented rabbits, although higher pulse energies were required to realise moderate effects with Nd:YAG as compared with diode laser irradiation. In albinos, visible laser burns were produced only by exposure to Nd:YAG laser energy. With both lasers and in pigmented and non-pigmented fundi, lesions were produced with an epicentre located within the retinal pigment epithelium.

The findings may be explained in terms of the relative beam tissue interaction characteristics of diode $(810 \mathrm{~nm})$ and Nd:YAG (1064 $\mathrm{nm})$ lasers. There are four principal components to this process - namely, the transmission, scatter, and absorption of radiation within the target tissue, and the morphological expression of their combined effects.

Transpupillary retinal irradiation is influenced by the transmission characteristics through the ocular media of the wavelength selected. Although transmission is high for both diode and Nd:YAG lasers, it is approximately $30 \%$ greater in the former than in the latter, ${ }^{1516}$ with a consequently higher irradiation at the retina for any given exposure (Table 1)

The intrinsic optical properties of a tissue will influence the degree of scattering and is, increased cross sectional diameter). The endothelium of the choriocapillaris was disrupted in some locations whereas in others it remained in situ, but in both instances, 
absorption and thus the distribution of radiant energy within it. Scatter increases with decreasing wavelength of incident radiation and also with increasing tissue thickness ${ }^{17}$; hence, for exposures in the near infrared region of the spectrum, it will be less than at visible wavelengths. In the presence of melanin, located within the pigmented retinal epithelium and choroid, the influence of primary absorption will dominate over that of photon scatter in inducing an absorption event. In the albino retina, which lacks melanin, internal scatter must assume a greater significance, with the increased pathlength of a scattered photon increasing the possibility of absorption occurring within the tissue.

Within the time domain between $100 \mathrm{~ms}$ to 10 second exposures, lesions are produced by photocoagulation. ${ }^{18}$ This process involves the absorption of radiant energy by a chromophore, and its conversion to heat by linear optical processes. The amount of energy absorbed by a pigment is a complex function of wavelength and energy of the incident radiation, as well as of pigment density and the pathlength over which the chromophore is distributed. ${ }^{19}$

The kinetics of coagulation are temperature and time dependent. A sustained temperature elevation of $10-20^{\circ} \mathrm{C}$ above the physiological level will result in disruption of enzymatic processes, protein denaturation, and the production of a threshold lesion. ${ }^{2021}$ If collagenous tissue is heated to $47^{\circ} \mathrm{C}$ above body temperature, collagen fibrils disintegrate into subfibrillar units. ${ }^{22} 23$ In practice, the peak temperature achieved at the centre of retinal lesions following photocoagulation may be in excess of $100^{\circ} \mathrm{C}^{2124}$ and at this level, collagen fibrils are no longer recognisable as discrete entities, a more or less homogeneous mass being produced.

Thermal diffusion from the centre of primary absorption will modify the damage profile of the lesion, this process being related to the density, specific heat and thermal diffusivity of the tissue, the rate of deposition of photon energy, and the peak temperature elevation. ${ }^{25}$

The chromophores which have been identified as the primary sites of absorption in retinal laser irradiation are melanin within the pigmented retinal epithelium and choroidal melanocytes and haemoglobin within the retinal and choroidal vasculature. Although intraretinal vessels are absent in the rabbit neuroretina, it is in the nature of infrared laser retinal photocoagulation that the principal zone of thermal destruction is centred upon the retinal pigment epithelium and inner choroid with some extension of tissue damage to the outer retina and mid choroid. Previous histological studies of diode laser irradiation of human retina have shown no evidence of laser related damage to vascular elements in the neurosensory retina. This would be predictable given a low absorption of any infrared radiation within haemoglobin. Although vascular flow may serve to dissipate any locally generated heat transients, again, previous studies appear to indicate that this would be a relatively minor effect. ${ }^{10}$ The higher absorption of $810 \mathrm{~nm}$ compared with $1064 \mathrm{~nm}$ radiation within melanin $^{2627}$ (Table 1) explains the difference in pulse powers required to produce moderately intense lesions.

The retinal damage profiles induced by diode and Nd:YAG laser exposures reflect the distribution of melanin within the pigmented retinal epithelium and choroid. The total amount of optical radiation absorbed within the choroid will exceed that dissipated by the pigmented retinal epithelium, owing to the larger total amount of melanin within the former. However, although the total pigmentation of the pigmented retinal epithelium is significantly less than that within the choroid, it is confined to a layer 5-10 $\mu \mathrm{m}$ thick, whereas in the choroid it may extend over $200-400 \mu \mathrm{m} .{ }^{9}$ Thus for any given exposure, the absorbed energy per unit pathlength is far greater in the pigmented retinal epithelium and consequently, higher temperatures are generated within this layer.

In the current investigation, the morphological appearance of burns corroborates these arguments: the primary site of damage was located within the pigmented retinal epithelium, with a meridionally deeper zone of damage being incurred by the underlying choroid. Changes observed in the overlying sensory retina may be ascribed principally to thermal flow from the pigmented retinal epithelium. The histological and ultrastructural observations are in agreement with those of other studies relating to diode laser retinal irradiation ${ }^{928} 29$ and Nd:YAG laser. ${ }^{30}$

That thermal damage was observed in albino rabbit retinas following exposure to Nd:YAG (but not diode) laser radiation suggests, naturally, the existence of an absorbing medium other than melanin. Another prerequisite appears to be a sufficiently high radiant exposure, given that a pulse energy of $10 \mathrm{~J}$ was required to produce visible lesions.

At high irradiances, haemoglobin within the blood of choroidal vessels could absorb sufficient energy to induce thermal damage, although the absolute values for absorption of near infrared radiation within this tissue are low $^{31}$ (Table 1). Initiation of haemoglobin denaturation induces changes in the absorptive properties of blood, this being related to the changes in light scattering properties and possibly also to an increase in intraluminal fluid density caused by evaporation. These factors may serve to enhance the process of intravascular absorption of infrared energy and thus increase the likelihood of thermal damage to adjacent structures. ${ }^{32-34}$ On the basis of this argument, the absence of blood flow stasis in the choriocapillaris was a somewhat surprising finding, given that the patterns of thermal damage observed in choroidal vessel walls and in the surrounding stroma, as well as in the retina, were otherwise similar to those realised in pigmented rabbits.

Another absorbing medium which may possibly be relevant to albino exposures is water. Radiation at $1064 \mathrm{~nm}$ is absorbed to a slight extent only in water and that at $810 \mathrm{~nm}$ to an even lesser degree ${ }^{35}$ (Table 1). However, at 
high irradiances, it is possible that the combination of a high photon density, with internal tissue scattering may be sufficient to induce absorption events even within water, such that a lesion is produced. Although the absorption of $810 \mathrm{~nm}$ radiation within blood is similar to that at $1064 \mathrm{~nm}$, its negligible absorption within water and the lower maximum power potential of the system afford an explanation for the lack of detectable lesions in albino retinas exposed to diode laser irradiation.

Although the lasers described are relatively recent developments, there is increasing interest in their clinical applications. Doubts have been raised, however, as to their suitability for retinal therapy, particularly in relation to their potential for inducing choroidal damage. ${ }^{36}$ However, the recanalisation of the choroidal vasculature which had occurred by 6 weeks indicates the reversible nature of the damage in this region. Clinical use of the diode laser in the treatment of diabetic retinopathy has failed to demonstrate any lasting adverse effects on the choroidal vasculature. ${ }^{13}$ It may also be argued that a system which has a relatively greater penetration into the choroid than occurs with shorter wavelength systems, ${ }^{28}$ may be more suitable for the treatment of choroidal lesions. Confirmatory evidence is provided by studies indicating the efficacy of diode laser photocoagulation in the treatment of choroidal neovascular membranes. ${ }^{37}$ The low absorption of infrared radiation within blood may also be of benefit in eyes with a haemorrhagic vitreous or preretinal opacities. Transmission will be higher than with the commonly used argon laser (emitting at $488-514.5 \mathrm{~nm}$ ) and therefore the potential effectiveness of retinal coagulation will be enhanced. Again, there is clinical evidence in support of this concept. ${ }^{13}$

In summary, the morphological effects of near infrared radiation upon retinal tissue are a consequence of scattering and absorptive events within melanin of the pigmented retinal epithelium and choroid in pigmented eyes and possibly within blood and water within the choroid of albino eyes. No evidence was found to contraindicate the clinical use of either laser and in certain situations their employment may have definite advantages.

This study was supported by grants from the Swiss National Science Foundation No 32.27748 .89 ) and the Swiss Commission for the promotion of Scientific Research (No 1835).

The authors are indebted to Wanda Giodano and Susanne Zimmerman for their technical expertise.

1 Meyer-Schwickerath G. Light coagulation (translated by SM Drance). St Louis: Mosby, 1960.

2 British Multicentre Study Group. Photocoagulation for proliferative diabetic retinopathy: a randomised controlled clinical trial using the xenon arc. Diabetologica 1984; 26: 109-15.

3 Maiman TH. Stimulated optical radiation in ruby. Nature 1960; 187: 493-4.

4 Taylor E. Regression of optic disc neovascularisation after retinal photocoagulation. Br f Ophthalmol 1970; 54: 535-9.

5 Diabetic Retinopathy Study Research Group. Photocoagulation treatment of proliferative diabetic retinopathy: the second report of diabetic retinopathy study findings. Ophthalmology (Rochester) 1978; 85: 82-1 05.

6 Hamilton AM. Management of diabetic retinopathy. Tran Ophthalmol Soc UK 1979; 97: 494-6.

7 Archer DB, Michalopoulos N. Treatment of neovascularisation secondary to branch retinal vein obstruction Int Ophthalmol 1981; 3: 141-53.
8 Macular Photocoagulation Study Group. Argon laser photocoagulation for senile macular degeneration: results of a randomised clinical trial. Arch Ophthalmol 1982; 100: 912-8.

9 McHugh JDA, Marshall J, Capon M, Rothery S, Raven A, Naylor PR. Transpupillary photocoagulation in the eyes of rabbit and human using a diode laser. Lasers Light Ophthalmol 1988; 2: 125-43.

10 McHugh JDA, Marshall J, ffytche TJ, Hamilton AM, Raven A. Macular photocoagulation with a diode laser. A comparative histopathological study. Lasers Light Ophthalmol 1990; 3: 11-28.

11 Van der Zypen E, Fankhauser F, Kwasniewska S, England C. Transpupillary irradiation of the irradiation with the cw-Nd.YAG laser. Invest Ophthalmol Vis Sci 1990; 31: $29-40$.

12 Van der Zypen E, Fankhauser F, England C, Kwasniewska $S$. Ultrastructure of the retina/choroid after irradiation with the cw-Nd.YAG laser. Lasers Light Ophthalmol 1990; 3: $277-80$.

13 McHugh JDA, Marshall J, ffytche TJ, Hamilton AM, Raven A, Keeler CR. Initial clinical experience using a diode laser in the treatment of retinal vascular disease. Eye 1989; 3: 516-27.

14 Okisaka S, Kashima K, Kimura T, Devaluti T, Nakajima A. Retinal photocoagulation using the Nd YAG laser. In: Docum Ophthal Proc Series 36. The Hague: Dr W Junk Publishers, 1984: 71-89.

15 Boettner EA, Wolter JR. Transmission of the ocular media. Invest Ophthalmol 1962; 1: 776-83.

16 Campbell CJ, Rittler MC, Innis RF, Shiner WH. Ocular effects produced by experimental lasers. III. Neodymium laser. Am F Ophthalmol 1968; 66: 614-32.

17 Parsa P, Jacques SL, Nishioka NS. Optical properties of rat liver between 350 and $2200 \mathrm{~nm}$. Appl Optics 1989; 28: 2325-30.

18 Ham WT, Ruffulo JJ, Mueller HA, Guerry D. The nature of retinal radiation damage. Dependence on wavelength, power level and exposure time. Vision Res 1978; 20: $1105-11$.

19 Gabel VP, Birngruber R, Hillenkamp F. Visible and near infrared light absorption in pigment epithelium and chorioid. In: Shimizu $\mathrm{K}$, ed. International congress Series No 450, XXIII Concilium Ophthalmologicum, Kyoto. Amsterdam-Oxford: Excerpta Medica, 1978: 658-62.

20 Mainster MA. Wavelength selection in macular photocoagulation. Ophthalmology 1986; 93: 952-6.

21 Van der Zypen E. The use of laser in eye surgery: morphological principles. Int Ophthalmol Clin 1985; 25: 21-52.

22 Mellerio J. The thermal nature of retinal laser photocoagulation. Exp Eye Res 1966; 5: 242-8.

23 Mainster MA, White TJ, Tips JH, Wilson PW. Retinal temperature increases produced by intense light sources. f Opt Soc Am 1970; 60: 264-70.

24 Jacques SL, Prahl SA. Modelling optical and thermal distributions in tissue during laser irradiation. Laser Surg Med 1987; 6: 494-503.

25 Watts GK. Biological effects of laser radiation on the eye. $\mathrm{PhD}$ thesis, 1970. London University.

26 Gabel VP, Birngruber R, Hillenkamp F. Individelle Unterscheide der Lichtabsorption am Augenhintergrund in Sichtbaren und infraroten Spectralbereich. Dtsch in Sichtbaren und infraroten Spectralbereich. Dtsch Ophthalmol

27 Wolbarsht ML, Walsh AW, George G. Melanin, a unique biological absorber. Appl Optics 1981; 20: $2184-6$

28 Brancato R, Pratesi R, Leoni G, Trabucci G, Vanni U. Histopathology of diode and argon laser lesions in rabbit retina. Invest Ophthalmol Vis Sci 1989; 30: 1504-10.

29 Smiddy W, Hernandes E. Histopathologic results of retinal diode laser photocoagulation in rabbit eyes. Arch Ophthalmol 1992; 110: 693-8.

30 Van der Zypen E, Fankhauser F, Kwasniewska S, England C. Transpupillary irradiation of the rabbit retina with the cw-Nd.YAG laser. Invest Ophthalmol Vis Sci 1990; 31: $29-40$.

31 Horeckler BL. The absorption spectrum of haemoglobin and its derivatives in the visible and near infrared regions. f Biol Chem 1943; 148: 173-8.

32 Fankhauser F, Kwasniewska S, van der Zypen E. Basic mechanisms underlying laser thrombogenesis in vascular structures of the eye. Lasers Light Ophthalmol 1989; 2: 223-31.

33 Van der Zypen E, England C, Fankhauser F, Kwasniewska $S$. Blood flow stasis induced by $\mathrm{cw}-\mathrm{Nd}$.YAG laser irradiation. Comparative morphology of mesenteric and choroidal vessels in

34 Van der Zypen E, Fankhauser F, Luscher EF, Kwasniewska $S$, England C. Induction of vascular haemostasis by Nd.YAG laser light in melanin-rich and melanin-free tissue. Doc Ophthalmol 1992; 79: 221-39.

35 Muller G, Dorschel K, Kar H. Biophysics of the ablation process. Lasers Med Sci 1991; 6: 241-54.

36 Gabel V-P, Lorenz B, Obana A, Vogel A, Birngruber R. Problems of clinical applications of diode lasers. Lasers Light Ophthalmol 1992; 4: 157-63.

37 Ulbig MRW, McHugh JDA, Hamilton AMP. Photocoagulation of choroidal neovascular lesions with a diode laser. Br 7 Ophthalmol 1993; 77: 218-22. 